\title{
In Honor of W.E. Moerner: Confining Molecules for Single-Molecule Spectroscopy
}

\author{
Adam E. Cohen, * Alexander P. Fields, Jennifer H. Hou, Sabrina R. Leslie, and Min Ju Shon \\ Departments of Chemistry and Chemical Biology and Physics, Harvard University, 12 Oxford Street, Cambridge, \\ Massachusetts 02138, USA
}

(Received 6 June 2009 and in revised form 9 July 2009

\begin{abstract}
Single-molecule spectroscopy provides a wealth of information on the dynamics and interactions of complex biological molecules. Yet these measurements are extremely challenging, partly because Brownian motion prevents molecules in free solution from remaining stationary. Here we describe several techniques that our lab has developed for confining single molecules for optical spectroscopy. These alternatives to surface immobilization provide confinement that is gentle enough to minimize perturbations to the molecule, but strong enough to allow long-time imaging of single fluorescent molecules, often in the presence of a high fluorescent background.
\end{abstract}

\section{INTRODUCTION}

This Special Issue celebrates a pioneer of single-molecule optical spectroscopy. From the early days of low temperature, high resolution, absorption spectroscopy, ${ }^{1}$ to modern fluorescence imaging of genetically modified proteins in living cells, ${ }^{2}$ W.E. Moerner has led the field by developing new methods for using light to probe single molecules. This article describes some tools our laboratory is developing, to further our shared longterm goal of watching single molecules wriggle and writhe in solution.

What information might one realistically hope to extract from a single molecule? Quantities of interest are largely the same as those probed in bulk biochemical assays, such as molecular size, shape, composition (stoichiometry), charge, response to mechanical stresses, binding affinities, and kinetics. Single-molecule tech- niques offer the hope to measure (1) multidimensional distributions that relate fluctuations in one quantity to fluctuations in another; and (2) time-dependent trajectories of molecules through this multidimensional state space. One might find, for instance, that a fluctuation in the charge of a molecule is followed after a short delay by a change in its binding kinetics.

Experimental challenges often interfere with the goal of simultaneous measurement of many molecular properties, molecule-by-molecule. The fundamental challenges are (1) that the photons detected from a molecule only contain indirect information about the state of the molecule, and (2) that molecules do not hold still in free solution for sufficient time to acquire good photon statistics or to observe slow molecular processes.

\footnotetext{
*Author to whom correspondence should be addressed. E-mail: cohen@chemistry.harvard.edu
} 
The light used in single-molecule spectroscopy typically has a wavelength in the visible region, spanning from 400 to $700 \mathrm{~nm}$. Most biomolecules, in contrast, have a diameter in the low single-digit nanometers: the median size of human proteins is 375 amino acids, corresponding to a diameter of $\sim 1.8 \mathrm{~nm}^{3}$ The diffraction limit of far-field imaging causes all molecules to appear as approximately Gaussian blobs, with an apparent lateral dimension of order half the wavelength of light. This mismatch between the wavelength of light and the size of most molecules precludes direct observation of the internal structure and dynamics of single molecules in all but a few cases. ${ }^{4,5}$ Even modern superresolution imaging schemes, such as STED, ${ }^{6}$ PALM,${ }^{7}$ and STORM,${ }^{8}$ have yet to achieve sub-molecular resolution. While direct imaging of the internal state is not yet possible for most molecules, the detected photons carry information in other channels, including their arrival times, polarizations, and wavelengths. The challenge of inferring molecular states from a series of observations on photons is a difficult one, and is unlikely to have a general solution.

The second challenge for single-molecule spectroscopy is that molecules in free solution jiggle and bounce like a class of kindergarteners. This Brownian motion causes the center of mass of a molecule to traverse a random walk, with a root-mean-square displacement

$$
d=\sqrt{2 D t}
$$

along each axis in time $t$, where $D$ is the diffusion coefficient. For spherical particles, the diffusion coefficient is given by the Stokes Einstein relation,

$$
D=\frac{k_{B} T}{6 \pi \eta r}
$$

where $k_{B} T$ is the thermal energy, $\eta$ is the viscosity, and $r$ is the radius of the particle. For non-spherical particles, $r$ may be replaced by an effective hydrodynamic radius which measures the average size of the particle. Smaller particles diffuse faster, and are therefore more difficult to study.

Single-molecule spectroscopic measurements handle Brownian motion in one of two ways. Some techniques observe freely diffusing molecules in bulk solution. Examples are Fluorescence Correlation Spectroscopy (FCS $)^{9}$ and its many variants, such as the Photon Counting Histogram $(\mathrm{PCH}),{ }^{10}$ Fluorescence Cross Correlation Spectroscopy (FCCS), ${ }^{11}$ and multi-focus FCS. ${ }^{12}$ These approaches avoid surface immobilization, but the observation time is typically $<1 \mathrm{~ms}$, limited by diffusion across a confocal observation volume. These freesolution techniques provide rather noisy quasi-static snapshots of molecules, limited by photon statistics and by the unknown diffusive trajectory the molecule takes through the observation volume. Additionally, these techniques are limited to low concentrations of fluorescent species; otherwise multiple molecules are likely to occupy the detection volume at the same time. This upper bound on the concentration prohibits measurements on weakly associating complexes, which are only found at high concentrations of their constituents. Confinement of the excitation volume via either total internal reflection (TIR) ${ }^{13}$ or zero-mode waveguides,,${ }^{14}$ allows measurements at higher concentration, but at the expense of even shorter diffusion-limited observation times.

The alternative strategy is to immobilize the molecules of interest on a surface, ${ }^{15}$ on a strand of DNA, ${ }^{16}$ on a cytoskeletal element, ${ }^{17}$ or inside a tethered lipid vesicle. ${ }^{18,19}$ Then, many single molecules can be imaged in parallel using wide-field fluorescence imaging, or individual molecules can be studied with high time resolution using confocal microscopy. These techniques have been applied particularly effectively in studies of molecular motors and nucleic acids and the proteins that bind to them. However, surface immobilization also suffers from drawbacks. Once a molecule is immobilized, it becomes difficult to learn about the size, shape, or charge of the molecule. Also, surface immobilization may disrupt the molecular behavior of interest.

Our lab takes a Goldilocks approach to molecular confinement: free molecules in 3-dimensional solution have too much Brownian motion; but rigid immobilization is overkill. Intermediate levels of confinement allow long-time observations of single-molecule dynamics, and at the same time maintain access to the geometrical and charge information encoded in transport coefficients. The ideal degree of confinement depends upon the experiment, so we have developed a suite of technologies for confining single molecules to varying extents.

Here we describe three such technologies. Figure 1 illustrates the principles underlying each technology, and Table 1 compares the new technologies with the more conventional schemes. Taken together, the new technologies allow one to observe single molecules without surface tethering, for longer times, at higher concentrations, and in greater number than was previously possible.

\section{ANTI-BROWNIAN ELECTROKINETIC TRAP (ABEL TRAP)}

The ABEL trap uses real-time particle tracking and electrokinetic feedback to suppress the Brownian motion of a single fluorescent molecule in solution at room 
(a)

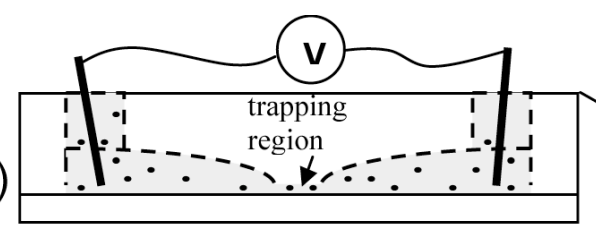

(b)

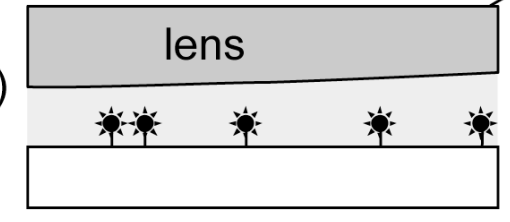

(c)

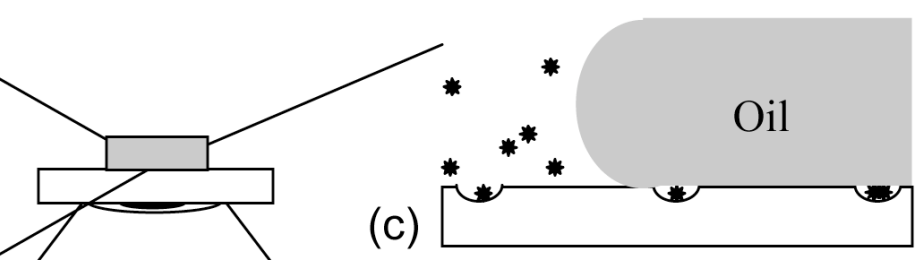

Fig. 1. Fluorescent imaging of single molecules, using a variety of nanostructured devices combined with an inverted fluorescence microscope. (a) The ABEL trap confines a single molecule to a small volume within a quasi two-dimensional trapping region. (b) The CLIC system generates a nanometer-scale film of solution in which to image either surface-tethered or freelydiffusing single molecules. (c) The dimple machine confines single molecules or molecular complexes into nanofabricated wells in a fused silica coverslip.

Table 1. Comparison of optical approaches to studying single molecules in free solution. Conventional methods experience a tradeoff between observation volume and observation time. The new techniques (in bold) avoid this tradeoff by using mechanical and electrical confinement to limit diffusion

\begin{tabular}{|c|c|c|c|c|}
\hline Imaging modality & $\begin{array}{l}\text { Dimensions of } \\
\text { imaging volume } \\
\mathrm{L} \times \mathrm{W} \times \mathrm{H}(\mu \mathrm{m})\end{array}$ & $\begin{array}{l}\text { Max concen- } \\
\text { tration of } \\
\text { single molecules }\end{array}$ & $\begin{array}{l}\text { Observation time } \\
\left(D=100 \mu \mathrm{m}^{2} / \mathrm{s}\right)\end{array}$ & $\begin{array}{l}\text { \# of molecules observed } \\
\text { simultaneously }\end{array}$ \\
\hline $\begin{array}{l}\text { Total Internal Reflection } \\
\text { Fluorescence (TIRF) }\end{array}$ & $100 \times 100 \times 0.1$ & $100 \mathrm{nM}$ & $50 \mu \mathrm{s}$ (vertical) & hundreds \\
\hline Confocal & $0.3 \times 0.3 \times 1$ & $10 \mathrm{nM}$ & $200 \mu$ s (in-plane) & 1 \\
\hline Zero-mode waveguides & $.04 \times .04 \times .02$ & $50 \mu \mathrm{M}$ & $2 \mu \mathrm{s}$ (vertical) & Thousands; 1 per waveguide \\
\hline ABEL trap & $3 \times 3 \times 0.8$ & $200 \mathrm{pM}$ & $2 \mathrm{~s}$ (photobleaching) & 1 \\
\hline $\begin{array}{l}\text { Convex Lens-Induced } \\
\text { Confinement (CLIC) }\end{array}$ & $100 \times 100 \times 0.005$ & $2 \mu \mathrm{M}$ & $25 \mathrm{~s}$ (in-plane) & hundreds \\
\hline Dimple machine & $.04 \times .04 \times .02$ & $50 \mu \mathrm{M}$ & $\begin{array}{l}\text { Limited by } \\
\text { photobleaching }\end{array}$ & Thousands; 1 per dimple \\
\hline
\end{tabular}

temperature. As a particle diffuses away from the target location, an electric field is applied to the solution to induce a combined electrophoretic and electroosmotic drift that returns the particle to the target location. For a more detailed discussion of the construction of the ABEL trap, the reader is referred to the primary literature and to a recent review. ${ }^{20-23}$ The trapping strength of the ABEL trap is limited by the speed and accuracy of the feedback loop.

Real-time tracking systems for single molecules fall into two categories: systems based on a camera and real-time image processing, and fully hardware systems. Early versions of the ABEL trap ${ }^{20,21,24}$ and a closely related system ${ }^{25,26}$ used wide-field epifluorescence illumination, high speed cameras, and computers to perform the tracking tasks. These systems have the advantage of flexibility and are easily reconfigured, but the feedback is typically too slow to trap objects smaller than approx- imately $20 \mathrm{~nm}$ in diameter in water. Smaller particles can be trapped by artificially increasing the viscosity of the trapping medium through the addition of glycerol or sucrose. Video-based ABEL traps have been used to immobilize and study individual virus particles, DNA molecules, CdSe quantum dots, and chaperonin proteins. ${ }^{27}$

An alternative approach uses hardware-based tracking, in which the photons are collected onto one or more photon-counting modules, and the timing or position of these photons is used to form an estimate of the location of the particle. ${ }^{22,28-33}$ Hardware tracking schemes can track the position of a particle photon by photon, achieving quantum-limited tracking bandwidth. However, hardware tracking is often sensitive to the alignment of the optics, and is difficult to reconfigure. Using a hardware trap, we trapped single chaperonin molecules in free solution, without artificially increasing the viscosity. We also partially suppressed the Brownian motion 
of single molecules of the fluorescent dye Cy3, yet did not stably trap the molecules. ${ }^{22}$

We recently developed an ABEL trap that combines the advantages of hardware- and software-based feedback systems. A pair of electro-optic beam deflectors moves a laser beam in a small confocal scan pattern at a scan rate as high as $80 \mathrm{kHz}$. The beam is directed into a microscope and illuminates a particle in the trap. The arrival time of each photon is used to update a Kalman filter that calculates an estimate of the location of a molecule. These calculations are performed in $2 \mu$ s on a commercial Field Programmable Gate Array (FPGA; National Instruments NI PCI-7831R), programmed via a LabView interface. This system runs with the speed of custom hardware, but the feedback parameters can be set in real time from the host PC.

Current incarnations of the ABEL trap provide feedback in two dimensions, with confinement in the third direction provided by a fused silica microfluidic cell. There is nothing, in principle, which prevents the ABEL trap from functioning in one or three dimensions as well. Such devices are currently under development. ${ }^{34}$

\section{Limitations to the ABEL trap}

The ABEL trap is ideally suited to study the conformation and dynamics of single objects with diameters in the range of $20-500 \mathrm{~nm}$, such as chaperonins or single molecules of DNA. The long observation times of multiply-labeled molecules permit detailed statistical analyses of the dynamics. However, the ABEL trap suffers from several drawbacks, namely: (1) a highly complex electro-optic system involving custom digital hardware and nanofabrication; (2) ability to trap only one molecule at a time, leading to low data throughput; (3) restriction to extremely dilute solutions $(<200 \mathrm{pM})$; and (4) need for high photon flux to maintain trapping, with correspondingly short lifetimes before photobleaching.

\section{CONVEX LENS-INDUCED CONFINEMENT (CLIC)}

In response to some of the limitations of the ABEL trap, we developed a system that confines molecules to a thin liquid film, where the film thickness varies continuously from zero up to several microns. Molecules are free to diffuse in the plane, but are confined in the vertical dimension. In its simplest incarnation, the CLIC system consists of a plano-convex lens, curved side down, resting on top of a coverslip. The region near the point of contact between the lens and the coverslip is imaged using an inverted fluorescence microscope. The lens-coverslip distance varies smoothly from zero at the point of contact, to hundreds of microns at points far from the point of contact, according to

$$
h \approx \frac{1}{2} \frac{r^{2}}{R}
$$

where $r$ is the distance from the point of contact and $R$ is the radius of curvature of the lens. Near the point of contact, a displacement of tens of microns in the $x-y$ plane leads to a nanometer-scale change in the thickness of the gap. Optical measurements at a series of radii spaced by microns lead to information on molecular properties at a series of confinements spaced by nanometers. In a typical field of view of $100 \mu \mathrm{m}$ centered on the point of contact, with a $100 \mathrm{~mm}$ focal length lens $(R=4.6 \mathrm{~cm})$, the gap varies from 0 to 27 nanometers. A detailed characterization of the CLIC system is in progress.

The CLIC system enables several new types of single-molecule measurements. These include (a) measurements on single immobilized molecules in the presence of a high background concentration of freely diffusing fluorescent molecules; (b) Long-time observation of single freely diffusing fluorescent molecules; and (c) direct mechanical measurements of molecular size and compressibility. Additionally, the CLIC system does not require any nanofabrication, sophisticated optics, electronics, or computer control. Applications of the CLIC system are discussed briefly below.

\section{Imaging in the presence of background fluorescence}

The focal volume probed by the CLIC system can be smaller in depth than that probed either by confocal or TIRF imaging. Single immobilized molecules can therefore be detected against a higher background concentration of fluorophores than in the other techniques. Zero-mode wave guides allow an even higher background concentration because of their confinement in all three dimensions, but such devices involve complex nanofabrication.

Figure 2 shows that the CLIC system provides imaging at higher signal-to-background than does throughthe-objective TIRF. Fluorescent beads of diameter 36 $\mathrm{nm}$ were immobilized on a coverslip and imaged in the presence of $50 \mathrm{nM}$ fluorescent dye (Alexa 647) in free solution. The figure shows the signal (bead fluorescence) relative to background (free dye fluorescence) as a function of displacement from the point of contact between the lens and the coverslip. Both through-theobjective TIRF (empty circles) and epifluorescence (filled circles) show increased signal-to-background near the point of contact. Remarkably, the signal-tobackground of epifluorescence near the point of contact is higher than that of TIRF far from the point of contact (where the lens has no effect). The CLIC system allows single-molecule imaging with high signal-to-background using a simple LED or lamp as the light source and an inexpensive objective, while TIRF requires laser illumination and an expensive oil-immersion objective with numerical aperture $>1.33$. 


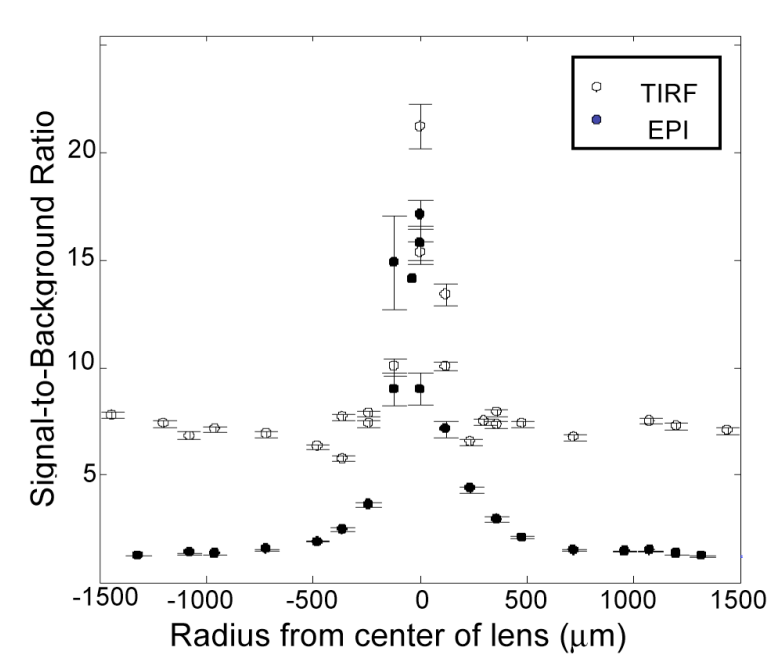

Fig. 2. The CLIC system provides better signal-to-background ratio near the region of contact than does either epifluorescence or TIRF illumination in the absence of confinement.

\section{Long-time observation of diffusing molecules}

Diffusion limits the time during which a single molecule in free solution may be observed. The diffusionlimited observation time is $\tau \approx d^{2} / 4 D$ if the molecule is diffusing in 2-D or $\tau \approx d^{2} / 2 D$ if the molecule is diffusing in 1-D. Here $d$ is the shortest linear dimension of the observation volume along which the molecule may escape. Table 1 shows that while zero-mode waveguides enable imaging at high concentrations, the tradeoff is an extremely short observation time for freely diffusing molecules ( $2 \mu \mathrm{s}$, typical). In TIRF imaging, small molecules diffuse out of the evanescent field in $\sim 200$ $\mu$ s, while in confocal imaging typical diffusion times are $\sim 500 \mu \mathrm{s}$. These short observation times place a severe constraint on the information that can be obtained from single freely diffusing molecules.

In the CLIC system, molecules are confined to a single focal plane in which they are free to diffuse. The tight vertical confinement prevents molecules from diffusing out of focus in the vertical direction and restricts the detection volume. In the horizontal direction, observation times are limited by the time for a molecule to diffuse across the field of view. For a typical setup with a field of view of $100 \mu \mathrm{m}$, and a small protein with a diffusion coefficient of $100 \mu \mathrm{m}^{2} / \mathrm{s}$, the observation time is $\sim 25 \mathrm{~s}$, an improvement of more than 4 orders of magnitude relative to confocal imaging. Stroboscopic illumination, synchronized to the frame-rate of the camera, can eliminate blurring of molecules on the timescale of an individual frame. ${ }^{35}$ Thus the CLIC system permits long-time observation of single molecules in free solution, in an extremely simple experimental geometry.

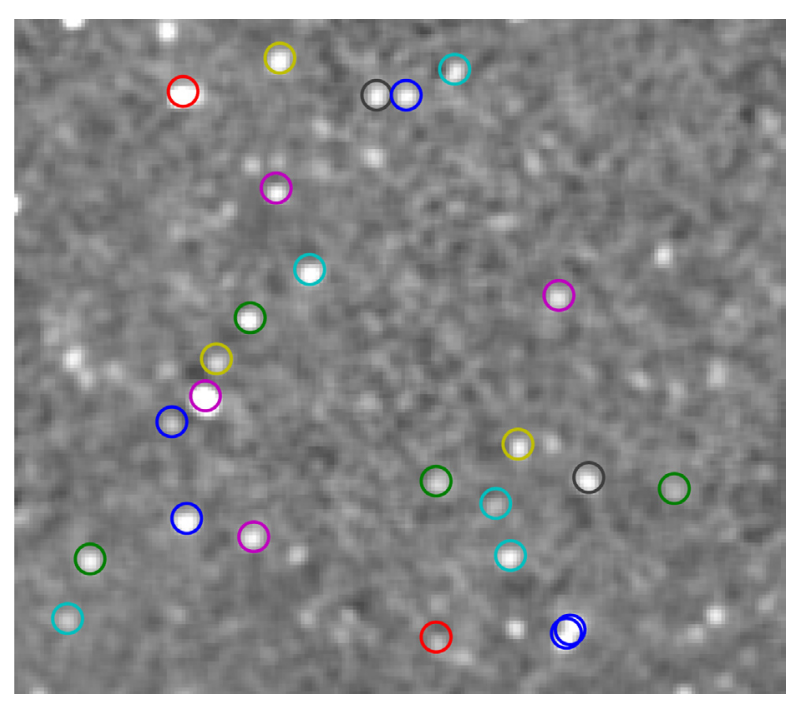

Fig. 3. Single mucin polymers confined in the CLIC system.

We performed proof-of-principle experiments in two biomolecular systems. In one experiment, we tracked single molecules of fluorescently labeled mucin polymers (extracted from pig gastric mucus), and compared their motion in a gel of unlabeled mucin to their motion in free solution (Fig. 3). These long-time tracking experiments revealed dynamical relaxation behaviors that would have been undetectable in shorter observations in unconfined systems. In a second experiment, we simultaneously tracked the motion of hundreds of fluorescently labeled molecules of $\lambda$-DNA. In both proofof-principle experiments we could track individual molecules for tens of seconds.

\section{Determining size and aspect ratio of freely diffusing particles}

The shallow wedge geometry of the CLIC system allows one to determine the size of freely diffusing macromolecular systems in two distinct ways. Molecules are excluded from the region where the thickness of the gap is less than the molecular diameter. A fluorescent image centered on the point of contact shows a disk inside of which there is no fluorescence (Fig. 4). From the radius of the excluded region, $r$, and the known radius of curvature of the lens, $R$, one can extract the diameter of the molecules, $h$. This measurement has an accuracy of $\sim 2$ $\mathrm{nm}$, and requires only $\sim 10 \mu \mathrm{L}$ of a $1 \mathrm{nM}$ solution.

An independent measure of molecular diameter is provided by the diffusion coefficient of molecules in the gap. Due to the long observation time, one can extract single-molecule diffusion coefficients with high accuracy. These diffusion coefficients are related to the 


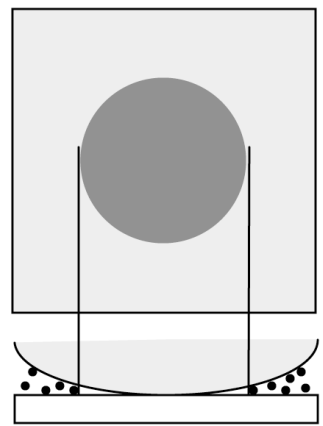

(a) (b)

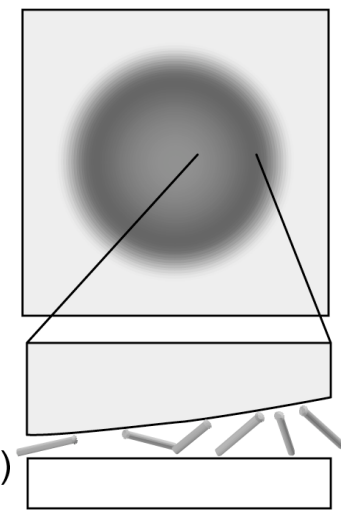

Fig. 4. (a) Determination of molecular size from the radius of the excluded region. (b) Determination of molecular aspect ratio or compressibility from the shape of the cutoff near the excluded region.

hydrodynamic radius via the Stokes-Einstein relation. There is a deviation from Stokes-Einstein behavior due to hydrodynamic interactions between the diffusing particle and the walls of the cell, but this effect can be corrected by imaging the diffusion of spherical polystyrene beads of known size.

The CLIC system also allows one to learn about the aspect ratio and compressibility of particles; information which would be very difficult to determine by conventional chromatographic means. For rodlike particles, there is an entropic penalty to enter the region where there is orientational confinement, but the particles are not completely excluded until the confinement is less than the diameter of the rod. By measuring the profile of particle density as a function of confinement, one can extract information about the length and aspect ratio of an asymmetric object. Such a technique is well suited to determine the size and shape of virus particles, for instance, or amyloid fibrils.

Flexible polymer molecules and lipid vesicles are also expected to have a gradual cutoff, even in a monodisperse population. Here the profile of the cutoff is determined by the entropic penalty of confining internal degrees of freedom. Thus one can, for instance, measure the free energy of a piece of DNA as a function of its confinement.

\section{Limitations to the CLIC system}

The CLIC system extends the range of conditions under which single molecules can be imaged, both for immobilized molecules and for molecules in free solution. However, the device requires that the lens and the coverslip be very clean and smooth. Asperities near the point of contact can lead to a non-zero distance of clos- est approach, and thereby to inaccuracies in the interpretation of data. Irregularities in the geometry of the lens or coverslip may be compensated by simultaneous imaging of a small molecule fluorophore in free solution, chosen to have a different color and not to interact with the molecules of interest. The brightness of this reference solution provides a measure of the gap thickness. In addition, the CLIC system is limited to imaging molecules tethered to a surface or in free solution; CLIC does not allow one to image the basal membrane of intact cells, for instance, while TIRF does.

\section{DIMPLE MACHINE}

To obtain detailed information on the internal dynamics of a single molecule one would like to hold the molecule still, without perturbing these dynamics. In such a case the in-plane Brownian motion of the CLIC system merely complicates the analysis. We designed a "dimple machine" to immobilize arrays of thousands of single molecules without surface attachment. The heart of the machine is a fused silica coverslip patterned with an array of nanofabricated dimples (Fig. 5). A solution of fluorescent molecules is washed over the dimples, and then replaced with an inert fluorocarbon oil. The oil traps a minute volume of solution in each dimple, containing at most a small number of molecules. The array of trapped molecules is imaged in a fluorescence microscope. The oil may be removed, and the process repeated an arbitrary number of times.

The dimple machine enables two new kinds of experiments. It is designed to probe (1) unimolecular dynamics without surface attachment; and (2) distributions of stoichiometries in multimeric complexes. In either case, tens of thousands of single molecules can be probed within a single minute, using either confocal or epifluorescence imaging.

To probe unimolecular dynamics, the dimples are bathed in a solution containing analytes at a concentration of less than one molecule per dimple, so that most dimples when capped contain either 0 or 1 molecule. Unimolecular dynamics can be probed via FRET, environmentally sensitive fluorophores, or polarization dynamics. The dimples need only be smaller than the resolution of the imaging system, so that the Brownian motion of the molecule within the dimple does not confound the measurement. After all the molecules have photobleached, the dimple array is refreshed and the measurement repeated. Examples of unimolecular measurements amenable to the dimple machine are probes of DNA cyclization, protein folding, and RNA folding.

The dimple machine also enables measurements of the distribution of stoichiometries in multimeric complexes. Consider the reaction $\mathbf{m} \mathbf{A}+\mathbf{n} \mathbf{B} \leftrightarrow \mathbf{A}_{\mathrm{m}} \mathbf{B}_{\mathrm{n}}$, where 

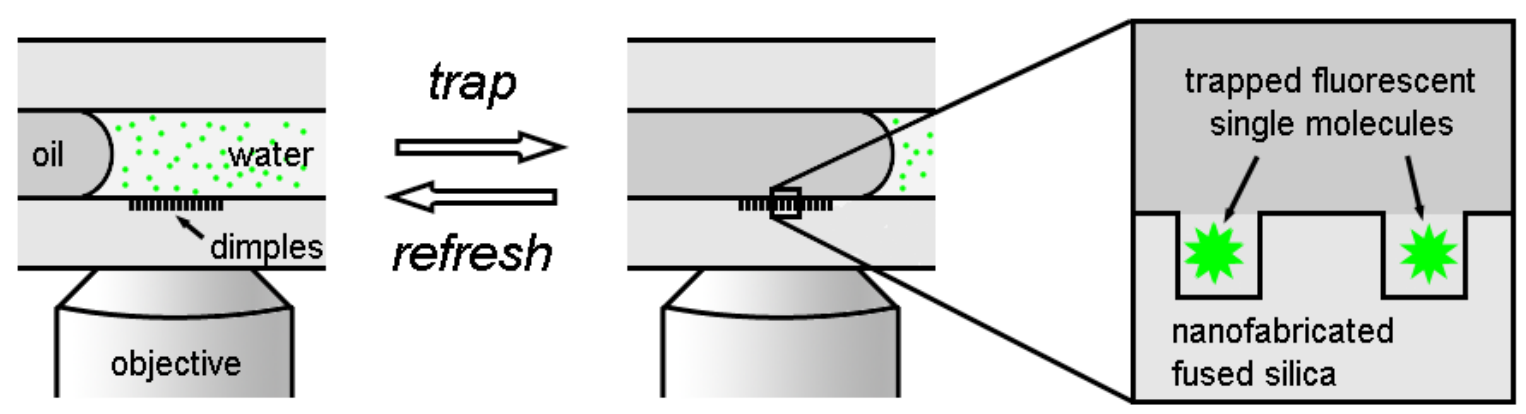

Fig. 5. Schematic layout of the dimple machine. A solution of fluorescent molecules is washed over an array of nanoscopic dimples fabricated in a fused silica coverslip. An inert oil is flowed over the dimples and traps a small number of molecules within each dimple.

$\mathbf{A}$ and $\mathbf{B}$ may be any pair of molecules, $\mathbf{n}$ and $\mathbf{m}$ may take on a range of values for multimeric complexes, and the distributions of $\mathbf{n}, \mathbf{m}$, and the associated equilibrium constant, $K_{e q}$, may be influenced by other components in the solution. Despite the simplicity of this reaction, there remains a need for a general-purpose tool that measures the distributions of $\mathbf{n}$ and $\mathbf{m}$ and the value of $K_{e q}$, particularly when the complex is weakly bound.

If species $\mathbf{A}$ and $\mathbf{B}$ are each labeled with a fluorescent dye of a different color, in principle one can measure the stoichiometry molecule-by-molecule, by measuring either the brightness or the number of photobleaching steps in each color. However, at the high concentrations needed to form weakly bound complexes, conventional single-molecule measurements may be impossible. The dimple machine isolates sub-wavelength volumes of solution, permitting single-molecule measurements at high concentrations of analyte. We are in the process of building a dimple machine in which the dimples have a diameter of only $30 \mathrm{~nm}$ and a depth of $30 \mathrm{~nm}$, i.e., with a volume of $2.7 \times 10^{-20} \mathrm{~L}$. At this dimple size, an analyte concentration of $60 \mu \mathrm{M}$ corresponds to on average 1 molecule/dimple.

\section{Limitations to the dimple machine}

The dimple machine enables single-molecule spectroscopy without surface tethering and at high concentrations. The chief limitations of the device are (1) the device requires sophisticated nanofabrication, and (2) the volume within each dimple is cut off from the bulk solution. Thus experiments that involve consumption of ATP or another small molecule may be hampered by the finite supply of the small molecule within each dimple.

\section{CONCLUSIONS}

It is unlikely that any single device will be optimal for all single-molecule fluorescence experiments. The ABEL trap permits long-time measurements on single molecules in free solution, and provides detailed information on diffusion coefficients and electrophoretic mobilities; but the ABEL trap is complex to operate, only probes one molecule at a time, and is restricted to extremely low concentrations. The CLIC system enables a wide variety of measurements on single molecules bound to surfaces or in free solution, but cannot reach to concentrations as high as are achieved by nanofabricated devices. The dimple machine gently immobilizes thousands of single molecules simultaneously, but provides no information on transport coefficients and requires complex nanofabrication. Ultimately all of these improvements to hardware are limited by the molecules being studied, i.e., the biochemical preparations and the stability of the fluorophores. Nonetheless, with proper confinement of single molecules, the future of single molecule spectroscopy is bright.

Acknowlegments: We thank Katharina Ribbeck for the mucin preparation. This work was partially supported by the National Science Foundation grant CHE-0910824 and the Materials Research Science and Engineering Center of the National Science Foundation under NSF Award DMR-02-13805.

\section{REFERENCES AND NOTES}

(1) Moerner, W.E.; Kador, L. Phys. Rev. Lett. 1989, 62, 2535.

(2) Biteen, J.S.; Thompson, M.A.; Tselentis, N.K.; Bowman, G.R.; Shapiro, L.; Moerner, W.E. Nature Methods 2008, 5, 947-949.

(3) Brocchieri, L.; Karlin, S. Nucleic Acids Res. 2005, 33, 3390.

(4) Cohen, A.E.; Moerner, W.E. Proc. Natl. Acad. Sci. USA 2007, 104, 12622.

(5) Yildiz, A.; Tomishige, M.; Vale, R.D.; Selvin, P.R. Science 2004, 303, 676-678.

(6) Hell, S.W. Far-Field Optical Nanoscopy. Science 2007, 316, 1153-1158.

(7) Betzig, E.; Patterson, G.H.; Sougrat, R.; Lindwasser, 
O.W.; Olenych, S.; Bonifacino, J.S.; Davidson, M.W.; Lippincott-Schwartz, J.; Hess, H.F. Science 2006, 313, 1642-1645.

(8) Rust, M.J.; Bates, M.; Zhuang, X. Nat. Methods 2006, 3, 793-795.

(9) Magde, D.; Elson, E.; Webb, W. Phys. Rev. Lett. 1972, 29, 705-708.

(10) Chen, Y.; Müller, J.D.; So, P.T.C.; Gratton, E. Biophys. J. 1999, 77, 553-567.

(11) Schwille, P.; Meyer-Almes, F.; Rigler, R. Biophys. J. 1997, 72, 1878-1886.

(12) Dertinger, T.; Pacheco, V.; von der Hocht, I.; Hartmann, R.; Gregor, I.; Enderlein, J. ChemPhysChem 2007, 8, 433-443.

(13) Axelrod, D. Traffic 2001, 2, 764-774.

(14) Levene, M.J.; Korlach, J.; Turner, S.W.; Foquet, M.; Craighead, H.G.; Webb, W.W. Science 2003, 299, 682686.

(15) Kuzmenkina, E.V.; Heyes, C.D.; Nienhaus, G.U. Proc. Natl. Acad. Sci. USA 2005, 102, 15471.

(16) Abbondanzieri, E.A.; Bokinsky, G.; Rausch, J.W.; Zhang, J.X.; Le Grice, S.F.J.; Zhuang, X. Nature 2008, 453, 184-189.

(17) Vale, R.D.; Milligan, R.A. Science 2000, 288, 88-95.

(18) Boukobza, E.; Sonnenfeld, A.; Haran, G. J. Phys. Chem. B 2001, 105, 12165-12170.

(19) Okumus, B.; Arslan, S.; Fengler, S.M.; Myong, S.; Ha, T. J. Am. Chem. Soc. 2009, 131, 14844.

(20) Cohen, A.E.; Moerner, W.E. Appl. Phys. Lett. 2005, 86,
093109.

(21) Cohen, A.E.; Moerner, W.E. Proc. SPIE 2005, 5699, 296.

(22) Cohen, A.E.; Moerner, W.E. Opt. Express 2008, 16, 6941-6956.

(23) Fields, A.P.; Cohen, A.E. Meth. Enzym, in press (2010).

(24) Cohen, A.E.; Moerner, W.E. Proc. Natl. Acad. Sci. USA 2006, 103, 4362-4365.

(25) Chaudhary, S.; Shapiro, B. IEEE Trans. Control Syst. Technol. 2006, 14, 669-680.

(26) Armani, M.; Chaudhary, S.; Probst, R.; Shapiro, B. J. Microelectromech. Sys. 2006, 15, 945-956.

(27) Cohen, A.E.; Moerner, W.E. Proc. Natl. Acad. Sci. USA 2006, 103, 4362-4365.

(28) Cang, H.; Wong, C.M.; Xu, C.S.; Rizvi, A.H.; Yang, H. Appl. Phys. Lett. 2006, 88, 223901.

(29) Xu, C.S.; Cang, H.; Montiel, D.; Yang, H. J. Phys. Chem. C 2007, 111, 32-35.

(30) Cang, H.; Xu, C.S.; Montiel, D.; Yang, H. Opt. Lett. 2007, 32, 2729-2731.

(31) Berglund, A.J.; Mabuchi, H. Optics Express 2005, 13, 8069-8082.

(32) McHale, K.; Berglund, A.J.; Mabuchi, H. Nano Lett. 2007, 7, 3535-3539.

(33) Berglund, A.J.; McHale, K.; Mabuchi, H. Opt. Lett. 2007, 32, 145-147.

(34) Davis, L. et al. Proc. SPIE 2008, 6862, 68620.

(35) Elf, J.; Li, G.W.; Xie, X.S. Science 2007, 316, 1191. 